\title{
Hipoacusia y deterioro cognitivo en adultos mayores
}

\author{
Hearing loss and cognitive impairment in the elderly
}

Jacobo A Aragón-Torres, ${ }^{*}$ Paula Weinberger Forische, ${ }^{*}$ Kelly Milla Hernández, ${ }^{\ddagger}$ Mónica Rodríguez-Valero§

\section{RESUMEN}

Antecedentes: Un tercio de los adultos mayores presentan hipoacusia, en México 67\% de las personas mayores de 60 años la padecen y en el Reino Unido 50\%. Pronto la hipoacusia será una de las enfermedades más frecuentes. Por otra parte, el deterioro cognitivo afecta a millones de personas, ocasionando discapacidad y dependencia. Se ha demostrado que la pérdida auditiva induce cambios en la función y estructura cerebral, acelerando el deterioro cognitivo. Objetivo: Describir la frecuencia de hipoacusia en los adultos mayores mexicanos con y sin diagnóstico de demencia y evaluar su asociación con el deterioro cognitivo. Material y métodos: Descripción de casos, adultos mayores de 65 años con Alzheimer, demencia no especificada y demencia, los controles fueron adultos mayores sanos. Pruebas aplicadas: Mini-Mental State Examination, Test de evaluación cognitiva de Montreal, audiometría tonal, Hearing Handicap Inventory for the Elderly-Screening. Análisis de datos: descriptivo, de asociación y cualitativo; identificando promedio, desviación estándar y porcentajes, utilizando SPSS. Prueba estadística: razón de momios. Resultados: Se estudiaron 10 casos y 17 controles. No existe diferencia estadísticamente significativa entre la edad, sexo, escolaridad, grado de educación y ocupación. La mitad de los pacientes con deterioro cognitivo presentaron una hipoacusia moderada a grave. Existe una asociación entre el grado de hipoacusia y el grado de deterioro cognitivo en el MMSE $(p=0.009)$, a mayor hipoacusia mayor deterioro cognitivo. Conclusiones: La hipoacusia es considerada un factor de riesgo modificable del deterioro cognitivo. En la población estudiada encontramos una alta frecuencia de deterioro cognitivo.

Palabras clave: Hipoacusia, déficit cognitivo, presbiacusia, adulto mayor.

Nivel de evidencia: III

\begin{abstract}
Background: One third of older people have hearing loss, in Mexico $67 \%$ of people over 60 have it, in the United Kingdom $50 \%$. Soon hearing loss will be one of the most frequent diseases. On the other hand, cognitive impairment affects millions of people, causing disability and dependence. It has been shown that hearing loss induces changes in brain function and structure, accelerating cognitive impairment. Objective: Describe the frequency of hearing loss in Mexican older people with and without a diagnosis of dementia and evaluate its association with cognitive impairment. Material and methods: Cases: adults over 65 with Alzheimer's disease, unspecified dementia and dementia. Controls: healthy older people. Tests: MiniMental State Examination, Montreal Cognitive Assessment, tonal audiometry, Hearing Handicap Inventory for the ElderlyScreening. Analysis: descriptive, association and qualitative; identifying average, standard deviation and percentages, using Statistical Package for the Social Sciences. Statistical test: odds ratio. Results: Ten cases and 17 controls were studied. There is no statistically significant difference between age, sex, schooling, education degree or occupation. Half of the patients with cognitive impairment presented moderate to severe hearing loss. There is an association between the degree of hearing loss and the degree of cognitive impairment in the MMSE $(p=0.009)$ a greater hearing loss is related to a greater cognitive impairment. Conclusions: Hearing loss is considered a modifiable risk factor for cognitive impairment. In the studied population was found a high frequency of cognitive impairment.
\end{abstract}

Keywords: Hearing loss, cognitive deficit, presbiacusia, elderly.

Level of evidence: III
* Facultad Mexicana de Medicina, Universidad La Salle. Ciudad de México.

‡ Escuela de Medicina, Universidad Westhill. Ciudad de México.

$\S$ Centro Neurológico, Centro Médico ABC. Ciudad de México.

Recibido para publicación: 22/09/2019. Aceptado: 29/11/2019.

Correspondencia: Dra. Mónica Rodríguez-Valero

Av. Carlos Graef Fernández Núm. 154, consultorio 156,

Col. Tlaxala Santa Fe, 05300, Cuajimalpa de Morelos, CDMX.

E-mail: dra.rodriguez.oi2@gmail.com

\section{Abreviaturas:}

CIE 10 = Clasificación Internacional de Enfermedades, 10ª

IMSS = Instituto Mexicano del Seguro Social.

MMSE $=$ Mini-Mental State Examination.

MoCA = Test de evaluación cognitiva de Montreal.

SPSS $=$ Statistical Package for the Social Sciences.

Este artículo puede ser consultado en versión completa en: www.medigraphic.com/analesmedicos 


\section{INTRODUCCIÓN}

El deterioro cognitivo es un síndrome clínico caracterizado por la disminución progresiva de las habilidades cognitivas y conductuales ocasionando discapacidad y dependencia, actualmente afecta a 46 millones de personas en el mundo. Sus principales causas son enfermedad vascular cerebral, trauma, infecciones, enfermedades metabólicas y neurodegeneración. ${ }^{1}$

Aproximadamente un tercio de los adultos mayores presentan algún grado de hipoacusia discapacitante. La causa más común de esta pérdida auditiva es la presbiacusia. La presbiacusia es un término que se aplica para describir la disminución de la audición relacionada con la edad y puede ser causada por muchos factores, entre los más frecuentes se encuentran la exposición a ruidos fuertes y antecedentes familiares. La presbiacusia se debe a una diminución de la función coclear, se ha descrito que con la edad se reduce el número de células ciliadas internas y las células del ganglio espiral. En otras palabras, el número de células funcionales de la cóclea disminuye con el paso del tiempo provocando una pérdida auditiva. ${ }^{2}$

La incidencia de pérdida auditiva en el adulto mayor es alta. En el Reino Unido ocho de cada 10 adultos mayores de 85 años tienen hipoacusia y la mitad de los adultos entre 60 y 85 años también presentan una pérdida auditiva. ${ }^{1}$ En la Ciudad de México $67 \%$ de los adultos mayores de 60 años sufren de algún grado de hipoacusia, porcentaje mayor que el reportado en el Reino Unido, ${ }^{3}$ mientras que la incidencia de hipoacusia en la población de 40 a 50 años es de $10 \%$. Se estima que en menos de 15 años, la hipoacusia será una de las 10 enfermedades más frecuentes en el adulto, siendo más común que la diabetes mellitus y las cataratas. ${ }^{1,4,5}$

En los últimos años se ha demostrado que la pérdida auditiva induce de manera directa o indirecta cambios en la función y estructura cerebral. ${ }^{6}$ También se ha reportado que la hipoacusia acelera el deterioro cognitivo. ${ }^{7}$ Por otro lado, los estudios que asocian la hipoacusia con el deterioro cognitivo han sido limitados a poblaciones caucásicas y su realización en población hispánica es rara, lo cual restringe el poder generalizar sus resultados, por lo que otro propósito de este estudio es aportar datos propios de nuestra población para futuras investigaciones.

El objetivo de este estudio retrospectivo de casos y controles es describir la frecuencia de la hipoacusia en población adulta mayor con diagnóstico de demencia y en población sin deterioro cognitivo diagnosticado. A su vez se evaluó si existe alguna asociación entre el grado de deterioro cognitivo y la presencia de hipoacusia en esta población.

\section{MATERIAL Y MÉTODOS}

Se llevó a cabo un estudio de casos y controles.

Casos: se invitó a participar a pacientes de la Clínica No. 19 del Instituto Mexicano del Seguro Social (IMSS). Los participantes se obtuvieron mediante solicitud al departamento de archivo bajo cualquiera de los siguientes diagnósticos según CIE 10: enfermedad de Alzheimer, demencia no especificada y demencia. Se incluyeron pacientes mayores de 65 años, cuya lengua materna fuera español con los diagnósticos de deterioro cognitivo previamente mencionados. Se excluyó a todo paciente que tuviera algún otro diagnóstico o enfermedad neurológica, enfermedad psiquiátrica, enfermedades otológicas, uso de auxiliares auditivos o que presentaran alguna alteración en el oído externo o medio durante la exploración física.

Controles: se invitó a participar a los acompañantes de los pacientes incluidos en los casos y a pacientes que acuden a la Clínica No. 19 del IMSS mayores de 65 años. En el caso de los pacientes con cita en la clínica sólo se incluyó a aquéllos que acudieran a valoración de otras patologías no relacionadas al área de neurología, psiquiatría u otorrinolaringología. Se excluyó a todos aquéllos que tuvieran algún antecedente de enfermedad neurológica, psiquiátrica u otológica.

Se firmó consentimiento informado de participación de los pacientes, familiares responsables y de los controles. Se hizo una historia clínica, exploración otorrinolaringológica y se evaluó la cognición a través del test de evaluación cognitiva de Montreal (MoCA) y Mini-Mental State Examination (MMSE).

Se realizó la audiometría tonal por examinador entrenado por conducción aérea de ambos oídos por medio de software SHOEBOX audiometry. Posteriormente se describe la audición de forma cualitativa (normal, leve, moderada, grave o severa-profunda).

La prueba de MMSE es una escala psicométrica breve que evalúa el estado cognitivo y consta de 30 ítems, la interpretación de la puntuación obtenida fue categorizada como deterioro cognitivo a aquéllos que presentaron un puntaje menor de 24 puntos, un puntaje de 30 determina un proceso cognitivo adecuado, un puntaje entre 25 y 30 representa un déficit ligero, un puntaje de 20-24 muestra un deterioro cognitivo leve, 15-19 puntos un deterioro cognitivo moderado y un puntaje menor de 14 corresponde a un deterioro cognitivo grave o demencia avanzada. El MoCA es una herramienta de cribado para iden- 
tificar la presencia de deterioro cognitivo y evalúa la atención, concentración, memoria de trabajo y de corto plazo, habilidades visoespaciales, fluidez verbal, lenguaje y funciones ejecutivas, con una puntuación máxima de 30 . Un puntaje de $0-25$ se interpreta como probable deterioro cognitivo y un puntaje mayor de 26 se considera normal. ${ }^{8,9}$

Se aplicó el cuestionario de discapacidad auditiva para el adulto mayor en su versión en español Hearing Handicap Inventory for the Elderly-Screening. ${ }^{10}$ Este instrumento fue validado en 1982 y evalúa el impacto emocional y social de la hipoacusia en los adultos mayores. Consiste en 13 preguntas que estudian las consecuencias emocionales, y 12 preguntas que miden las consecuencias sociales y situacionales de la hipoacusia. Tiene una buena reproducibilidad y niveles aceptables de sensibilidad y especificidad para detectar personas con hipoacusia clínicamente significativa. Los resultados se evalúan desde 0 (ninguna desventaja) a 40 (desventaja máxima). ${ }^{11}$

Se realizó un análisis estadístico descriptivo y de asociación entre la presencia de hipoacusia y la presencia de deterioro cognitivo. Los datos se analizaron mediante el Statistical Package for the Social Sciences (SPSS) versión 21. Las variables y los datos fueron analizados descriptiva y cualitativamente de forma inicial, identificando el promedio, la desviación estándar y porcentajes. Las pruebas estadísticas utilizadas fueron razón de momios.

\section{RESULTADOS}

Se estudiaron 10 casos y 17 controles. Los casos tuvieron una edad promedio de $80.7 \pm 7.8$ años y los controles $75.3 \pm 6.3$ años, $80 \%$ de los casos fueron mujeres y $52 \%$ de los controles también fueron mujeres. La mitad de los casos y de los controles tuvieron estudios hasta la primaria (completa e incompleta). $20 \%$ de los casos y $29 \%$ de los controles tienen un trabajo. No existe diferencia estadísticamente significativa entre la edad, sexo, escolaridad, grado de educación y ocupación (Tabla 1). El grupo de los pacientes con diagnóstico de deterioro cognitivo presentaron un puntaje en el MMSE y en el MoCA menor que los controles $(15.9 \pm 7$ vs. $25.4 \pm 2$ y $11.5 \pm 7$ vs $19.9 \pm 4$ respectivamente, $\mathrm{p}<0.001$ ). Llama la atención que todos los sujetos evaluados, tanto los controles como lo casos, excepto una paciente, revelaron resultados alterados en el MoCA, mientras que en el MMSE 58\% de los controles muestran resultados que sugieren algún grado de deterioro cognitivo (Tabla 2). En cuanto a la audición, todas las hipoacusias encontradas
Tabla 1: Características demográficas.

\begin{tabular}{lcc}
\hline & $\begin{array}{c}\text { Casos } \\
\mathrm{N}=10\end{array}$ & $\begin{array}{c}\text { Controles } \\
\mathrm{N}=17\end{array}$ \\
\hline Edad & $80.7 \pm 7.8$ & $75.3 \pm 6.3$ \\
Mujeres & $8 / 10$ & $9 / 17$ \\
Escolaridad & & \\
$\quad$ Primaria incompleta & 2 & 3 \\
$\quad$ Primaria & 3 & 5 \\
$\quad$ Secundaria & 1 & 1 \\
$\quad$ Preparatoria & 0 & 0 \\
$\quad$ Licenciatura o postgrado & 4 & 8 \\
Ocupación & & \\
$\quad$ Hogar & 5 & 5 \\
$\quad$ Empleo & 2 & 5 \\
$\quad$ Jubilado/a & 2 & 5 \\
Desempleo & 0 & 2 \\
\hline
\end{tabular}

fueron del tipo sensorineural. Existe una diferencia de $10 \mathrm{~dB}$ en los umbrales auditivos de ambos grupos. Dentro de los controles 54\% tuvo una disminución en la audición leve, $5 \%$ presentó una audición adecuada y $17 \%$ grave. Más de la mitad de los pacientes con deterioro cognitivo mostraron una hipoacusia moderada a grave. En el grupo de los controles más de la mitad de los sujetos presentan una audición normal o una pérdida leve en comparación con los casos en los que sólo $20 \%$ está en esta categoría. La hipoacusia neurosensorial moderada y grave fue más común en el grupo de los casos. 92\% de la población evaluada mostró algún grado de hipoacusia, 10\% de los casos y $5 \%$ de los controles revelaron una audición adecuada.

Existe una asociación entre el grado de hipoacusia y el grado de deterioro cognitivo en el MMSE ( $p=$ 0.009), a mayor hipoacusia mayor deterioro cognitivo.

De la misma manera según el cuestionario autoadministrado Hearing Handicap Inventory for the Elderly la media del puntaje fue de 13.4 y 26 para los controles y los casos, el cual en relación con la disminución en la cognición tuvo significancia estadística $(\mathrm{p}=0.021)$.

\section{DISCUSIÓN}

La demencia es un problema de salud global, con el incremento en la longevidad el deterioro cognitivo ya es considerado un problema de salud pública significativo. Se estima que el número de personas que viven con demencia en 2015 será triplicado para 2050. En 2017 Lancet posicionó la pérdida auditiva como el mayor factor de riesgo modificable de demencia junto con otros nueve factores de salud y estilo de vida. 
La pérdida auditiva por edad o presbiacusia es muy frecuente, afecta hasta $40 \%$ de la población mayor de 65 años. En nuestro estudio observamos una prevalencia mayor, tanto en la población sana (presente en 94\%) como en los pacientes con demencia (presente en $90 \%$ ). Se ha descrito que hasta $9 \%$ de los casos de deterioro cognitivo están relacionados con hipoacusia. ${ }^{12}$

La pérdida auditiva es un factor de riesgo del deterioro cognitivo modificable, por lo que su estudio ha ganado relevancia en los últimos años. Múltiples estudios afirman esta relación y han encontrado un grado de asociación variable. ${ }^{13}$ La pérdida auditiva acelera el deterioro cognitivo por ser una deprivación sensorial, desencadenar aislamiento social y aumentar la carga cognitiva; también se ha descrito una etiología patológica compartida de ambas patologías. La hipoacusia en el adulto mayor se correlaciona con una pérdida de volumen cerebral en la corteza auditiva, en el lóbulo temporal y de la masa encefálica global. ${ }^{6}$ La presbiacusia, a su vez se considera un marcador de fragilidad en esta población ${ }^{14}$ y se relaciona con mayor esfuerzo auditivo y con una reducción en la reserva cognitiva. ${ }^{13}$ Loughrey demostró que la presbiacusia se ha asociado específicamente con el deterioro de la memoria episódica y con la velocidad de procesamiento cognitivo. ${ }^{15}$ La hipoacusia también lleva a un aislamiento social y se asocia con depresión, ambos factores contribuyen a un deterioro cognitivo acelerado. ${ }^{16}$ Los estudios de cohorte muestran que la presbiacusia precede de cinco a 10 años al deterioro cognitivo, por lo que la pérdida auditiva empieza a considerarse como un posible biomarcador no invasivo. También sobresale que los pacientes con pérdida auditiva tienen un deterioro $54 \%$ más rápido en el MMSE que los pacientes normoacúsicos. ${ }^{5}$ La asociación significativa entre pérdida auditiva y demencia también ha sido descrita en población mexicana por Estrada-González. ${ }^{17}$ En 2011 Lin describe que por cada $10 \mathrm{~dB}$ perdidos en la audición hay una reducción de cinco puntos en el MMSE. También concluyeron que el deterioro cognitivo asociado a una pérdida de $25 \mathrm{~dB}$ en la audición equivale al deterioro cognitivo que ocurre en el lapso de siete años. ${ }^{18}$

La deprivación sensorial, ya sea hipoacusia o diminución en la agudeza visual es común en personas con deterioro cognitivo. Además de acelerar la progresión del deterioro también exacerba la discapacidad y la carga de los cuidadores. La pérdida auditiva deteriora la comunicación efectiva con los cuidadores provocando un deterioro en la calidad de vida.

En nuestro estudio sobresale que un alto porcentaje de los controles presenta un puntaje alterado en las pruebas cognitivas. Encontramos una alta inci- dencia de algún grado de deterioro cognitivo en nuestros controles evaluados con MoCA y sólo 58\% con el MMSE. En relación con el Mini-Mental, el MoCA analiza una gama más amplia de funciones cognitivas (con un mayor énfasis en la función ejecutiva) y tiene elementos más difíciles. Una de las principales virtudes del MoCA es que identifica mejores estados preclínicos como el deterioro cognitivo leve, ya que incluye dominios no evaluados por el Mini-Mental como el funcionamiento ejecutivo, la atención y el recuerdo diferido. ${ }^{9}$ La discrepancia entre los resultados de ambas pruebas en el grupo control, la atribuimos a que el MoCA detecta el deterioro cognitivo leve y de forma más temprana. ${ }^{19}$

Hasta el momento no existen tratamientos efectivos que modifiquen el deterioro cognitivo; sin embargo, el retraso de la aparición del deterioro tiene grandes implicaciones positivas tanto en lo individual, en lo colectivo y en la economía. El tratamiento de la pérdida auditiva puede potencialmente retrasar o prevenir hasta un tercio de los casos de demencia. Rehabilitar la audición en adultos (45-65 años) es la mejor intervención que se puede hacer para prevenir o retrasar la aparición de este deterioro cognitivo. ${ }^{16}$ La rehabilitación auditiva consiste en mejorar la calidad de la audición para mejorar la comprensión del lenguaje a través del uso de auxiliares auditivos, amplificadores de sonidos, implantes de oído medio e implantes cocleares. Los efectos de la rehabilitación auditiva con auxiliares auditivos e implantes cocleares en el desarrollo o progresión del deterioro cognitivo son variables. ${ }^{20}$ Estudios demuestran que el uso de auxiliares auditivos mejora los índices de calidad de vida en pacientes con hipoacusia y Alzheimer. ${ }^{21}$ Lin

Tabla 2: Descripción de resultados cognitivos y auditivos.

\begin{tabular}{lcc}
\hline & Casos & Controles \\
$\mathrm{N}=10$ & $\mathrm{~N}=17$
\end{tabular}

MoCA = test de evaluación cognitiva de Montreal; $\mathrm{dB}=$ decibeles . 
describe que las personas con hipoacusia rehabilitadas con auxiliares auditivos tienen un mejor desempeño en las pruebas cognitivas que las personas con hipoacusia no rehabilitada. ${ }^{18}$ En cuanto a pacientes con implantes cocleares hay evidencia de que los usuarios de implantes cocleares con deterioro cognitivo leve muestran una disminución en la progresión del deterioro. ${ }^{22}$

El deterioro cognitivo es un padecimiento que cada vez es más frecuente y uno de los factores de riesgos modificables es la hipoacusia. Hoy en día la pérdida auditiva es la discapacidad con mayor número de soluciones, desde auxiliares auditivos hasta implantes de tallo cerebral. Numerosos estudios demuestran las consecuencias cognitivas de no tratar la pérdida auditiva de manera temprana, por lo que es de suma importancia rehabilitar la audición en adultos para así prevenir el desarrollo y retrasar el deterioro cognitivo.

Debido a que éste es un estudio piloto, la principal limitación de nuestro estudio es el número de sujetos estudiados. Este factor influye probablemente en la alta frecuencia de deterioro cognitivo en población sana.

\section{CONCLUSIÓN}

La hipoacusia y el deterioro cognitivo son dos padecimientos que son más comunes en la población de edad avanzada. La hipoacusia es considerada un factor de riesgo modificable del deterioro cognitivo. La hipoacusia moderada y grave es más frecuente en la población con deterioro cognitivo diagnosticado. En la población estudiada encontramos una alta frecuencia de deterioro cognitivo.

\section{BIBLIOGRAFÍA}

1. Dawes P, Cruickshanks K, Moore D, Edmondson-Jones M, McCormack A, Fortnum H et al. Cigarette smoking, passive smoking, alcohol consumption, and hearing loss. JARO. 2014; 15 (4): 663-674.

2. Sergeyenko Y, Lall K, Liberman MC, Kujawa SG. Age-related cochlear synaptopathy: an early-onset contributor to auditory functional decline. J Neurosci. 2013; 33 (34): 13686-13694.

3. Pedraza-García Z, Delgado-Solís M. El déficit de audición en la tercera edad. Rev Fac Med UNAM. 2008; 51: 91-95.

4. Global costs of unaddressed hearing loss and cost-effectiveness of interventions: a WHO report, 2017. Geneva: World Health Organization; 2017. Licence: CC BY-NC-SA 3.0 IGO.

5. Gurgel RK, Ward PD, Schwartz S, Norton MC, Foster NL, Tschanz JT. Relationship of hearing loss and dementia: a prospective, population-based study. Otol Neurotol. 2014; 35 (5): 775-781.
6. Lin F, Ferrucci L, An Y, Goh J, Doshi J, Metter E et al. Association of hearing impairment with brain volume changes in older adults. Neuroimage. 2014; 90: 84-92.

7. Selkoe D, Hardy J. The amyloid hypothesis of Alzheimer's disease at 25 years. EMBO. 2016; 8 (6): 595-608.

8. Loureiro C, García C, Adana L, Yacelga T, RodríguezLorenzana A, Maruta C. Uso del test de evaluación cognitiva de Montreal (MoCA) en América Latina: revisión sistemática. iNeuro. 2018; 66 (12): 397.

9. Aguilar-Navarro S, Mimenza-Alvarado A, Palacios-García A, Samudio-Cruz A, Gutiérrez-Gutiérrez L, Ávila-Funes J. Validez y confiabilidad del MoCA (Montreal Cognitive Assessment) para el tamizaje del deterioro cognoscitivo en México. Revista Colombiana de Psiquiatría. 2018; 47 (4): 237-243.

10. Lichtenstein M, Hazuda H. Cross-cultural adaptation of the hearing handicap inventory for the elderly-screening version (HHIE-S) for use with Spanish-Speaking Mexican Americans. J Am Geriatr Soc. 1998; 46 (4): 492-498.

11. Ventry IM, Weinstein BE. The hearing handicap inventory for the elderly: a new tool. Ear Hear. 1982; 3: 128-134.

12. Dawes P. Hearing interventions to prevent dementia. HNO. 2019; 67 (3): 165-171.

13. Hardy CJ, Marshall CR, Golden HL, Clark CN, Mummery CJ, Griffiths TD et al. Hearing and dementia. J Neurol. 2016; 263 (11): 2339-2354.

14. Panza F, Solfrizzi V, Seripa D, Imbimbo BP, Capozzo R, Quaranta $\mathrm{N}$ et al. Age-related hearing impairment and frailty in Alzheimer's disease: interconnected associations and mechanisms. Front Aging Neurosci. 2015; 7: 113.

15. Loughrey DG, Kelly ME, Kelley GA, Brennan S, Lawlor BA. Association of age-related hearing loss with cognitive function, cognitive impairment, and dementia: a systematic review and meta-analysis. JAMA Otolaryngol Head Neck Surg. 2018; 144 (2): 115-126.

16. Livingston G, Somerlad A, Orgeta V, Costafreda SG, Huntley $\mathrm{J}$, Ames D et al. Dementia prevention, intervention, and care. Lancet. 2017; 390: 2673-734.

17. Estrada-González JG, Morales-Cadena GM, Dorado-Berumen OA, Fonseca-Chávez MG. Estado funcional y cognitivo de los adultos mayores relacionado con el grado de hipoacusia. An Orl Mex. 2018, 63 (1): 11-14.

18. Lin FR. Hearing loss and cognition among older adults in the United States. J Gerontol. 2011; 66 (10): 1131-1136.

19. Ciesielska N, Sokołowski R, Mazur E, Podhorecka M, Polak A, K $\square$ dziora K. Is the Montreal Cognitive Assessment (MoCA) test better suited than the Mini-Mental State Examination (MMSE) in mild cognitive impairment (MCI) detection among people aged over 60? Meta-analysis. Psychiatr. 2016; 50: $1039-1052$.

20. Uchida Y, Sugiura S, Nishita Y, Saji N, Sone M, Ueda H. Agerelated hearing loss and cognitive decline - The potential mechanisms linking the two. Auris Nasus Larynx. 2019; 46 (1): $1-9$.

21. Dawes P, Wolski L, Himmelsbach I, Regan J, Leroi I. Interventions for hearing and vision impairment to improve outcomes for people with dementia: a scoping review. Int Psychogeriatr. 2019; 31 (2): 203-221.

22. Mosnier I, Vanier A, Bonnard D, Lina-Granade G, Truy E, Bordure $\mathrm{P}$ et al. Long-term cognitive prognosis of profoundly deaf older adults after hearing rehabilitation using cochlear implants. J Am Geriatr Soc. 2018; 66 (8): 1553-1561. 